\title{
Prediction of the Lifetime of Nitrile-Butadiene Rubber by FT-IR
}

\author{
Tetsuya KaWASHIMA ${ }^{* \dagger}$ and Toshio OGaWA** \\ *Nitto Analytical Techno-Center Co., Ltd., 1-1-2, Shimohozumi, Ibaraki, Osaka 567-8680, Japan \\ **Laboratory for Ecological Polymer Chemistry, Kanazawa Institute of Technology, \\ 7-1, Ohgigaoka, Nonoichi, Ishikawa 921-8501, Japan
}

\begin{abstract}
A quantitative measurement method with FT-IR was proposed for a thermal degradation analysis of nitrile-butadiene rubber (NBR). An NBR film was prepared as a model sample on a barium fluoride $\left(\mathrm{BaF}_{2}\right)$ crystal plate, which was subjected to a heat treatment. The absorbances of various functional groups were measured directly by FT-IR after thermal degradation at high temperatures. By measuring the absorbances, it was possible to readily determine quantitatively each of the functional groups after the degradation of NBR. By assuming that the NBR lifetime was the point at which the absorbance of a carbon-carbon double bond reaches $45 \%$ of that prior to thermal treatment, a method for predicting the lifetime of NBR heated below $150^{\circ} \mathrm{C}$ was proposed, by using an Arrhenius plot of the heating time versus heating temperature.
\end{abstract}

(Received July 19, 2005; Accepted September 14, 2005)

\section{Introduction}

In recent years, electronic devices using flexible printed circuit (FPC) boards have come to be used in a wide range of fields, such as automobiles and mobile phones. The conditions for using these devices have changed rapidly. The devices that have been used indoors under constant temperatures will have to be sufficiently reliable, even when used at high temperature and under high humidity. Elastomers can often be used as a blend with thermosetting adhesives composed of epoxy resin or other resins in order to improve the impact resistance and the flexibility. For example, elastomer blended epoxy resins are widely used in FPC boards as adhesives for affixing polyimide films and patterned copper foil circuits. The blending of elastomer mitigates external stress and generates novel characteristics, such as flexural resistance, and impact resistance, which are not attainable with epoxy resins alone. ${ }^{1}$

The present authors have carried out research on the degradation of adhesives composed of nitrile rubber (NBR)/epoxy resin adhesive that were used in FPC. ${ }^{2-4}$ Adhesive layers on FPC boards serve not only to affix polyimide films and patterned copper foil, but also to insulate the patterns with each other. ${ }^{2}$ Copper migrates into the adhesive when FPC is used at high temperature under high humidity. The copper ions liberated from the pattern eventually form copper oxides with impurity ions, and selectively precipitate in NBR, which forms a microphase-separated structure. ${ }^{3}$ It has been confirmed that the migration of copper ions leads to a dramatic decrease in the insulation resistance between patterns. In addition, when FPC was used at high temperature under high humidity, a decrease in the glass transition temperature $\left(T_{\mathrm{g}}\right)$ of the adhesive was recognized; the decrease in the osmic acid staining capacity was

† To whom correspondence should be addressed.

E-mail: tetsuya_kawashima@gg.nitto.co.jp observed by a transmission electron microscope (TEM). ${ }^{4}$ These studies suggested that the degradation of adhesives was caused by the degradation of NBR.

The degradation of NBR and other diene rubbers can be roughly classified into 3 categories: thermal degradation, ${ }^{5,6}$ photo degradation, ${ }^{7,8}$ and ozone degradation. ${ }^{9}$ According to these references, polymers undergo primary chain cleavage due to peroxy radical reactions, leading to a decrease in the molecular weight and the generation of various new functional groups in the molecules through oxidation reactions. When FPC boards and other such materials are used as automotive parts, the degradation of NBR in adhesives is strongly related to thermal factors.

In this paper, a direct quantitative analysis of the contents of several functional groups in NBR, such as a carbon-carbon double bond and a nitrile group under heating by a Fourier Transform Infrared Spectrophotometer (FT-IR), is described. From an accelerated lifetime test at over $150^{\circ} \mathrm{C}$ and a measurement of the absorbance of degraded NBR after heating, a method for predicting the lifetime of NBR heated below $150^{\circ} \mathrm{C}$ is also proposed.

\section{Experimental}

The sample polymer was nitrile-butadiene rubber (NBR) in which a medium-high amount of acrylonitrile was compounded with a small amount of acrylic acid copolymerized as a third monomer. The samples used in FT-IR measurements were prepared as follows: after $0.5 \mathrm{~g}$ of NBR was dissolved in $10 \mathrm{~mL}$ of chloroform, the solution was added dropwise to barium fluoride $\left(\mathrm{BaF}_{2}\right)$ crystal plates (diameters $13 \mathrm{~mm}$, thickness 2 $\mathrm{mm}$ ), and the plates were allowed to dry. The film thickness of the NBR film on the plates prepared under these conditions was found to be approximately $3 \mu \mathrm{m}$ by measuring the cross section of the film by scanning electron microscopy (SEM). $\mathrm{BaF}_{2}$ 


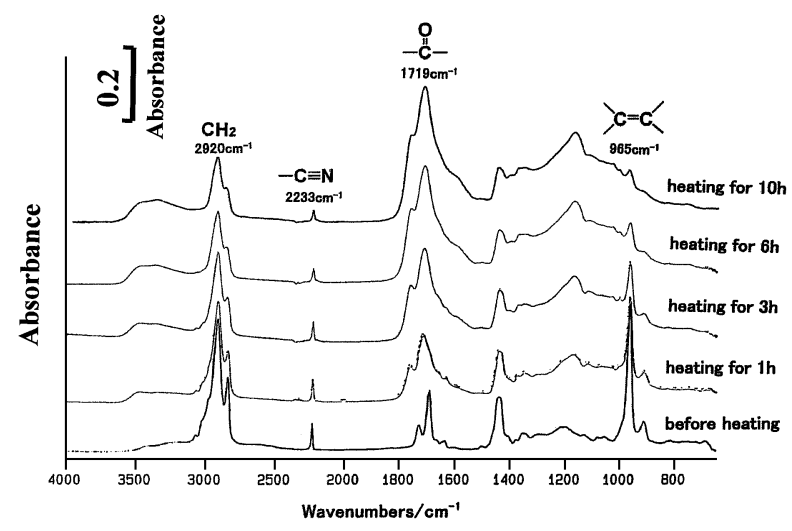

Fig. 1 FT-IR spectra of NBR samples before and after heating at $200^{\circ} \mathrm{C}$.

crystal exhibits almost no absorption in the range of the FT-IR measurement, and is thermostable over $500^{\circ} \mathrm{C}$ or higher..$^{10}$

The NBR film on the $\mathrm{BaF}_{2}$ crystal plate was placed in an electric furnace (FM-35R, Yamato) at a definite period and temperature, and the sample crystal plate was removed for an FT-IR measurement. The sample plate was brought back to the furnace for a heat treatment at another definite period under the same temperature after the FT-IR measurement. The heat treatment and FT-IR measurements were repeated until the total time of the heat treatment of the sample plate in the furnace reached total $10 \mathrm{~h}$.

Infrared spectra were measured using a Bio-Rad Fouriertransform infared spectrophotometer (Model FTS-45). The measurements were taken at a resolution of $8 \mathrm{~cm}^{-1}$ with a scanning number of 64 . Thermally treated and untreated NBR films prepared on $\mathrm{BaF}_{2}$ crystal plates were used for measurements.

\section{Results and Discussion}

There are several techniques of FT-IR measurements: transmission spectroscopy, internal reflection spectroscopy (often called attenuated total reflection spectroscopy (ATR)), external reflection spectroscopy, diffuse reflectance spectroscopy, photoacoustic spectroscopy, and emission spectroscopy. In all of these techniques, the transmission technique has several advantages: ${ }^{11}$ the highest signal-to-noise ratio when the sample has the proper thickness, no sampling technique convolution of the spectra, and easy quantification. Transmission spectroscopy can be used for several samples forms: solutions, films, and powder as $\mathrm{KBr}$ pellets or in Nujol. When polymers are dissolved in a solvent, the transmitted infrared spectra have a disadvantage concerning a disturbance by a infrared absorption caused from the solvent used. The $\mathrm{KBr}$ pellet method of polymer sampling should be used with caution because the technique involves the use of tremendous pressures, which could seriously affect the sample. Transmission spectroscopy is primarily useful if uniform thin films of polymeric samples are available.

When quantitative analysis is carried out using infrared spectra, the absorbance should be normalized with respect to a chemical structurally invariable absorbance. ${ }^{12}$ In the present work, however, the normalization technique was not employed, because our method used the same sample disk for the same sample polymer when infrared absorption spectra of the
Table 1 Lifetime of NBR

\begin{tabular}{lrrrrrrr} 
Temperature $/{ }^{\circ} \mathrm{C}$ & 150 & 180 & 190 & 200 & 220 & 230 & 250 \\
Heating time $/ \mathrm{h}$ & 54.7 & 10.3 & 5.0 & 1.9 & 1.7 & 0.72 & 0.20 \\
\hline
\end{tabular}

Note: The lifetime is designated as the time at which the absorbance of the $\mathrm{C}=\mathrm{C}$ group $\left(965 \mathrm{~cm}^{-1}\right)$ attains $45 \%$ of the initial value.

designated sample polymer were measured by changing the heating time, and a direct comparison of the absorption intensities was possible, thus eliminating any possible changes in the absorbance due to a difference in the film thickness. Figure 1 shows the infrared absorption spectra of NBR obtained by this technique, where the change with the heating time in the infrared absorption spectra of NBR at $200^{\circ} \mathrm{C}$ is presented. The baseline between 4000 and $3700 \mathrm{~cm}^{-1}$ of all spectra in Fig. 1 is indicated as absorbance 0 .

The absorbance of the carbon-carbon double bond (1,4 transbonding, $\left.965 \mathrm{~cm}^{-1}\right)$ and the nitrile group $\left(2233 \mathrm{~cm}^{-1}\right)$ changed with the heating time. In addition, the absorbance of hydroxyl groups at $3350 \mathrm{~cm}^{-1}$ and $3470 \mathrm{~cm}^{-1}$ and that of the carbonyl group at $1719 \mathrm{~cm}^{-1}$ increased with the heating time. Moreover, the extremely broad carbonyl group absorption band at 1719 $\mathrm{cm}^{-1}$ may indicate that the absorption band of carboxylate ester $\left(1729 \mathrm{~cm}^{-1}\right)$ or $\alpha, \beta$-unsaturated ketone $\left(1690 \mathrm{~cm}^{-1}\right)$ contributes to this absorption band. The appearance of a shoulder peak at $1763 \mathrm{~cm}^{-1}$ was also observed, which is thought to be attributed to the carbonyl group absorption as a result of the generation of carboxylic acid at a carbon bonded to the nitrile group. ${ }^{12}$

A thermal treatment of polyacrylonitrile results in an internal cyclization reaction of a nitrile group (polyimide formation), followed by dehydrogenation to form a highly conjugated structure. ${ }^{5}$ An immediate decrease (consumption) in the nitrile group $\left(2233 \mathrm{~cm}^{-1}\right)$ indicates cyclization, and a strong decrease in aliphatic hydrogens $\left(2920 \mathrm{~cm}^{-1}\right.$ and $\left.1450 \mathrm{~cm}^{-1}\right)$ suggests concurrent aromatization. The rapid growth of a band at 1600 $\mathrm{cm}^{-1}$ is attributed to imide formation (cyclization). The thermal degradation of NBR produces several functional groups, such as carbonyl and carboxyl groups, due to oxidation reactions, as mentioned above. An investigation of this degradation has been carried out by the qualitative and quantitative analyse for these degradation products caused by oxidation.

An accelerated test of the sample polymer was carried out by changing the temperatures from $150^{\circ} \mathrm{C}$ to $250^{\circ} \mathrm{C}$, and the changes in the absorbance with the heating time were determined. The results for several functional groups are shown in Figs. 2 (a) to (d) for the carbon-carbon double bond (965 $\left.\mathrm{cm}^{-1}\right)$, the methylene group $\left(2920 \mathrm{~cm}^{-1}\right)$, the nitrile group (2233 $\left.\mathrm{cm}^{-1}\right)$, and the carbonyl groups $\left(1719 \mathrm{~cm}^{-1}\right)$, respectively.

The abundance of the carbon-carbon double bond, the nitrile group and the methylene group was found to decrease with the heating time, and the decreasing rate of the carbon-carbon double bond was the fastest at each temperature, followed by the nitrile group and the methylene group. The carbon-carbon double bond decreased to $10 \%$ or less in approximately $1 \mathrm{~h}$ at $250^{\circ} \mathrm{C}$. On the other hand, the abundance of the carbonyl group in NBR increased remarkably with the thermal treatment. For example, after a treatment for $1 \mathrm{~h}$ at $250^{\circ} \mathrm{C}$, it increased by approximately 5 -fold abundance before the thermal treatment.

An accelerated lifetime test at $120^{\circ} \mathrm{C}$ under $100 \%$ relative humidity for NBR/epoxy resin adhesives revealed that the glass transition temperature of these adhesives is depressed by as much as approximately $10^{\circ} \mathrm{C}$ after a $300 \mathrm{~h}$ treatment. ${ }^{4}$ An accelerated lifetime test of NBR was also carried out under the 


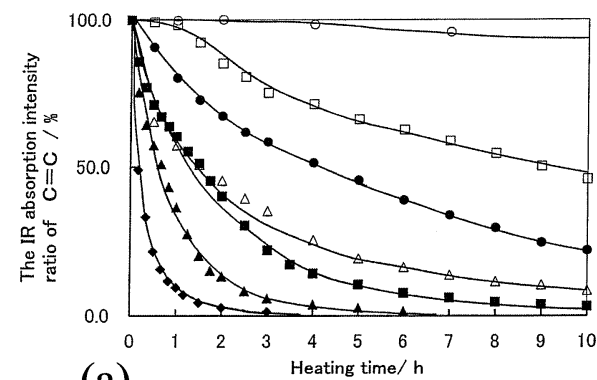

(a)

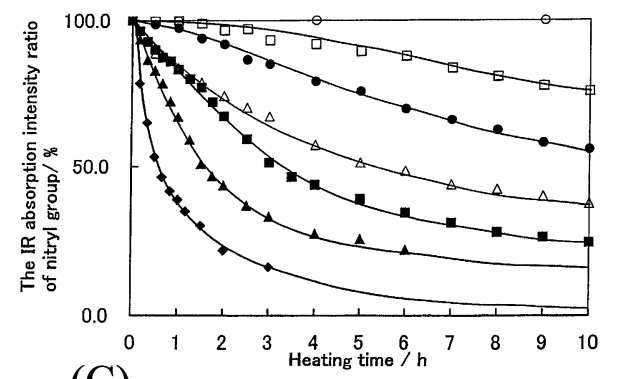

(C)

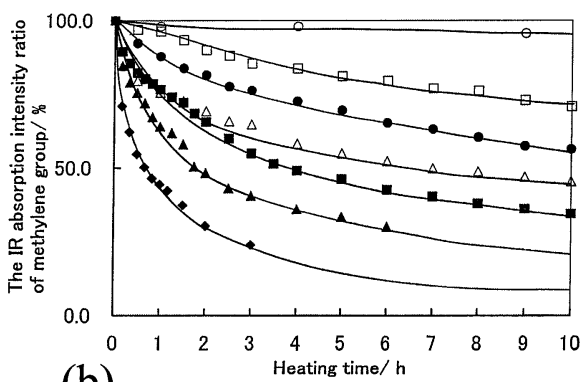

(b)

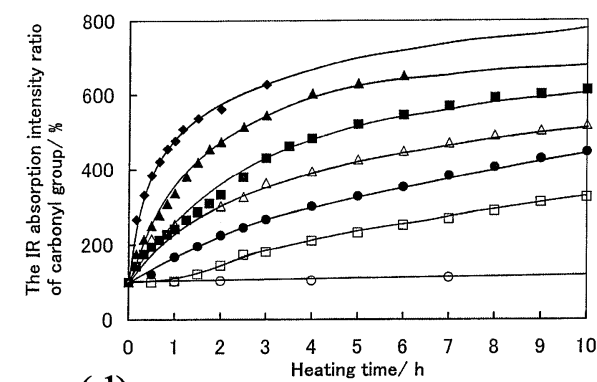

(d)

Fig. 2 Absorbance ratios of several functional groups compared with those at a heating time of $0 \mathrm{~h}$ against the heating time (h) at different heating temperatures. (a) C-C double bond $\left(965 \mathrm{~cm}^{-1}\right)$; (b) methylene group $\left(2920 \mathrm{~cm}^{-1}\right)$; (c) nitrile group $\left(2233 \mathrm{~cm}^{-1}\right)$; (d) carbonyl group $\left(1719 \mathrm{~cm}^{-1}\right), \mathrm{O}$, $150^{\circ} \mathrm{C} ; \square, 180^{\circ} \mathrm{C} ; \bullet, 190^{\circ} \mathrm{C} ; \triangle, 200^{\circ} \mathrm{C} ; \boldsymbol{\square}, 220^{\circ} \mathrm{C} ; \boldsymbol{\Delta}, 230^{\circ} \mathrm{C} ; \bullet, 250^{\circ} \mathrm{C}$.

same conditions, and the absorbance ratio of the carbon-carbon double bond with respect to the methylene group was 0.686 when the glass transition temperature reached below $10^{\circ} \mathrm{C}$ from the original one. In the present investigation, the absorbances of various functional groups were measured at several temperatures, and it was found that when the absorbance ratio of the carbon-carbon double bond with the methylene group reached 0.686 , the absorbance of the carbon-carbon double bond was approximately $45 \%$ of the initial value. This point can be considered as the NBR degradation point (lifetime). By increasing the heating temperature, the heating time attained at the point where the absorbance of the carbon-carbon double bond became $45 \%$ from the initial value decreased, as listed in Table 1.

The lifetime of NBR can be predicted by using the Arrhenius life prediction method based on reaction kinetics. The Arrhenius equation is expressed as follows:

$$
k=A^{-\mathrm{E} / \mathrm{RT}}
$$

where $A$ denotes the frequency factor, $E$ is the activation energy, $k$ denotes the rate constant, $R$ denotes the gas constant, and $T$ is the absolute temperature. Taking the logarithm of both sides of Eq. (1) gives

$$
\ln k=-E / R T+\ln A \text {. }
$$

Because the terms $\ln k$ and $1 / T$ thus have a linear relationship, the activation energy can be determined from the slope of this line. ${ }^{13}$ If the change in the absorbance of the carbon-carbon double bond can be approximated by a function of the reciprocal of the thermal treatment time, as shown in Fig. 2(a), the rate constant $(k)$ can be substituted for the reciprocal of the heating time (thermal treatment time, $t(h))$. Thus, when the function of $\ln (1 / t)$ and $1 / T$ is plotted, the activation energy $(E)$ can be obtained as the slope. The Arrhenius plot of the heating temperatures and the heating times at the point of $45 \%$ absorbance of the carbon-carbon double bond (the point at which the $T_{\mathrm{g}}$ of the NBR/epoxy resin adhesive is depressed by approximately $10^{\circ} \mathrm{C}$ ) gave a good linear relationship using the data of Table 1 as

$$
\ln (1 / t)=-11.9 \times 1000(1 / T)+24.2 .
$$

And the activation energy was calculated to be $23.6 \mathrm{kcal} / \mathrm{mol}$ from the slope. Converting this data to a function of time, $t(\mathrm{~h})$, and temperature $T(\mathrm{~K})$, the following equation was obtained:

$$
t=1 / \exp (-11.9 \times 1000 / T+24.2)
$$

The term $t$ can be estimated as the lifetime of NBR after heating for $t \mathrm{~h}$. Though Eq. (4) was obtained by an accelerated lifetime test between $150^{\circ} \mathrm{C}$ and $250^{\circ} \mathrm{C}$, it may be possible to predict the lifetime of NBR below $150^{\circ} \mathrm{C}$. The calculated heating temperature and lifetime below $150^{\circ} \mathrm{C}$ is as follows: lifetime of $50 \mathrm{~h}$ at a heating temperature of $150^{\circ} \mathrm{C}$; lifetime of $2200 \mathrm{~h}$ at $100^{\circ} \mathrm{C}$; lifetime of $1.3 \times 10^{4} \mathrm{~h}$ at $80^{\circ} \mathrm{C}$; lifetime of $3.1 \times 10^{5} \mathrm{~h}$ at $50^{\circ} \mathrm{C}$.

\section{Conclusions}

NBR/epoxy resin adhesives used in FPC boards degrade when subjected to high temperatures; this degradation depends on that of NBR, which forms a micro-phase separated structure. A technique was devised for quantitatively determining these changes, using an NBR thin film formed directly on a $\mathrm{BaF}_{2}$ crystal plate, and measuring the samples before and after degradation by FT-IR. By measuring the absorbances, it was possible to readily quantitatively determine each of the 
functional groups. The carbon-carbon double bond, the nitrile group, and the methylene group decreased and the carbonyl group in NBR increased with the thermal treatment. The rates of the decrease and the increase were more rapid at high temperature. It is possible to assume that the NBR lifetime was the point at which the absorbance of the carbon-carbon double bond reached $45 \%$ of that prior to thermal treatment, where the glass transition temperature of NBR was depressed by $10^{\circ} \mathrm{C}$. From the Arrhenius plot of the heating time and the heating temperature at the point of $45 \%$ absorbance of the carbon-carbon double bond, the relationship of the lifetime of NBR and the temperature can be obtained. Based on this degradation life prediction method, the life of NBR is dramatically shortened at temperatures exceeding $100^{\circ} \mathrm{C}$.

(Part of this paper was presented at the 4th Symposium of Macromolecular Analysis 1999, November Nagoya.)

\section{References}

1. I. Shibata and Y. Hibino, Circuit Tech., 1988, 3, 296.

2. T. Ogawa, A. Kuratani, S. Osawa, and H. Inoue, Materiel Life, 1998, 10, 85.
3. T. Kawashima, T. Sugimoto, and T. Ogawa, Materiels Life, 1999, 11,71 .

4. T. Kawashima and T. Ogawa, Bunseki Kagaku, 2000, 49, 835

5. M. Celima, D. K. Ottesen, K. T. Gikken, and R. L. Clough, Polym. Degrad. Stab., 1997, 58, 15.

6. G. Ahlblad, K. Jacobson, and B. Stenberg, Plast. Rubber Composit. Proces. Appl., 1996, 25, 464.

7. C. Adam, J. Lacoste, and J. Lemaire, Polym. Degrad. Stab., 1990, 27,85 .

8. X. Jouan, C. Adam, D. Fromageot, J. Gardette, and J. Lemaire, Polym. Degrad. Stab., 1989, 25, 247.

9. T. Hara, T. Ogawa, S. Osawa, S. Nakamura, S. Yoshida, and K. Minegishi, Materiels Life, 1998, 10, 93.

10. K. Miyagawa and M. Tsuboi, in "Kagaku Daijiten 7 (Encyclopedia Chimica, in Japanese)", ed. Chemical Dictionary Committee, 1964, Kyouritsushuppan, Tokyo, 877.

11. J. L. Koenig, "Spectroscopy of Polymers", 1992, ACS, Washington, D.C., 46.

12. B. Antczak and B. Krajewska, Int. Polym. Sci. Technol., 1984, 11,46 .

13. K. Hirota and K. Kuwata, "Kagaku Dourikigaku (Chemical Kinetics, in Japanese)”, 1971, Kyoritsushuppan, Tokyo, 52. 